Relations industrielles

Industrial Relations

\title{
Les centrales syndicales et le problème du chômage
}

\section{Jacques St-Laurent}

Volume 16, numéro 2, avril 1961

URI : https://id.erudit.org/iderudit/1021802ar

DOI : https://doi.org/10.7202/1021802ar

Aller au sommaire du numéro

Éditeur(s)

Département des relations industrielles de l’Université Laval

ISSN

0034-379X (imprimé)

1703-8138 (numérique)

Découvrir la revue

Citer ce document

St-Laurent, J. (1961). Les centrales syndicales et le problème du chômage.

Relations industrielles / Industrial Relations, 16(2), 238-242.

https://doi.org/10.7202/1021802ar

Tous droits réservés (C Département des relations industrielles de l’Université Laval, 1961
Ce document est protégé par la loi sur le droit d'auteur. L’utilisation des services d'Érudit (y compris la reproduction) est assujettie à sa politique d'utilisation que vous pouvez consulter en ligne.

https://apropos.erudit.org/fr/usagers/politique-dutilisation/ 


\section{COMMENTAIRES}

\section{Les centrales syndicales et le prob!ème du chômage}

JaOques St-Laurent

1. Depuis quelques mois, le Congrès du Travail du Canada (CTC) et la Confédération des Syndicats Nationaux (CSN) ont eu plusieurs fois l'occasion de présenter aux autorités fédérales leurs vues sur le problème du chômage et sur la ou les façons de l'éliminer.

Le CTC a présenté trois documents principaux: le premier à la conférence sur le chômage, tenue à Ottawa, les 24 et 25 octobre dernier; le deuxième, au gouvernement canadien le 2 février et le troisième, au comité du sénat chargé d'étudier les problèmes d'emploi et de maind'oeuvre, le 8 février. La CSN, à notre connaissance, n'a présenté à date (le 10 mars 1961) que deux documents: l'un à la conférence sur le chômage, l'autre au cabinet fédéral.

Dans l'ensemble, tous ces documents posent les mêmes problèmes et offrent à peu près les mêmes solutions. Ils se distinguent surtout parce qu'ils ne sont pas tous d'égale qualité et parce que l'importance accordée aux problèmes et leurs solutions varient beaucoup.

Le meilleur de tous ces documents est sans contredit le mémoire du CTC au sénat canadien. C’est aussi le plus volumineux. Pour connaître la pensée du CTC sur le problème du chômage, on peut y reférer et délaisser ses deux autres mémoires qui ne sont que le résumé de celui-ci.

2. Il est assez curieux de noter que les deux centrales ne semblent pas envisager de la même façon les institutions économiques dans lesquelles on vit. Il semble que les dirigeants du CTC acceptent plus facilement ces institutions mais prétendent qu'on ne les utilise pas de la meilleure façon possible. Les dirigeants de la CSN, eux, vont beaucoup plus loin. Ils affirment même que «si l'on persiste, consciemment ou non, à vouloir nous démontrer que la liberté d'entreprise équivaut à chômage, c'est l'entreprise libre qui disparaîtra ». ${ }^{1}$ Cette affirmation catégorique disparaît cependant d'un second mémoire de la CSN. On y retrouve là une attitude plus conciliante. On prend, par exemple, le soin de noter que la proposition sur la création d'un conseil économique «ne mérite pas l'anathème qu'on a prononcé contre elle» et que \& des

(1) Mémoire de la CSN, à la Conférence sur le chômage, Ottawa, 25 oct. 1961, p. 2. 
conseils semblables existent dans plusieurs pays dont l'économie est dominée par l'entreprise privée ». ${ }^{2}$ En dernière analyse, il semble donc que les positions des deux centrales ouvrières vis-à-vis le système économique actuel ne diffèrent pas tellement entre elles - ou est-ce une illusion?

3. Une autre caractéristique des mémoires de la CSN, c'est qu'on ne s'y débarrasse pas facilement d'énoncés de principes du genre de celui qui suit: "l'homme ne vit pas de pain seulement et, si nous ne sommes en mesure de créer une société dans laquelle il pourra satisfaire tous ses besoins d'homme, nous aurons failli à notre tâche commune ». ${ }^{3}$ Puis, tout le reste du mémoire traite des mesures à prendre pour assurer au travailleur le pain quotidien... En général, les mémoires du CTC sont plus «matter of fact» et ne s'embarrassent pas d'énoncés de principes.

4. Dans le mémoire des uns et des autres, on accorde une importance toute particulière au chômage causé par autre chose que les cycles (saisonniers ou autres) ou le degré d'immobilité de la main-d'oeuvre (le chômage frictionnel). Ce qui inquiète les dirigeants du travail, c'est le développement croissant du nombre de chômeurs qui demeureraient chômeurs même si on réussissait à faire disparaître les cycles saisonniers, les récessions et les frictions. Ce qui les inquiète, c'est le problème chronique du chômage de longue période. «The recession unemployment is the most immediate problem... but it is by no means the biggest problem. There is a second, even bigger. Even if we get back to where we were last year... we shall still have an unemployment problem of frightening proportions... this is the problem of long-run chronic unemployment »." * Jusqu'ici, on nous parlait de chômage saisonnier, jusqu'à un certain point, on avait raison... Tel n'est plus le cas cependant $\gg .{ }^{5}$

Le chômage chronique est devenu la préoccupation majeure des dirigeants syndicaux. Ce chômage n'est plus particulier à certaines régions canadiennes. Il menace de devenir une caractéristique de notre économie.

5. Le CTC s'est attaqué à ce problème avec beaucoup de précision. ${ }^{6}$ Le Congrès soutient que ce problème en est un de croissance économique. Le nombre de situations offertes n'augmente pas au rythme de l'augmentation dans la main-d'oeuvre. Pour rétablir l'équilibre, il ne faut pas réduire l'offre de travail au niveau de la demande, mais porter cette dernière au niveau de la première. Si le secteur privé laissé à lui-même est dans l'impossibilité de le faire, — et il semble bien que:

(2) Mémoire de la CSN, au cabinet fédéral, 2 février 1961, p. 3.

(3) Id., p. 1.

(4) CTC. Mémoire à la conférence fédérale sur le chômage, octobre 1961, p. 3.

(5) CSN. Idem.

(6) V. surtout son mémoire au comité du Sénat. 
ce soit le cas - que le gouvernement intervienne par des investissements massifs. Financés de quelle façon? Par une hausse des taxes? Non pas. Cela ne ferait que transférer les dépenses d'un secteur à l'autre, sans les augmenter. Par la vente au public d'obligations du gouvernement? Non plus, puisque cela aurait le même effet. Mais par un déficit financé à même la création de monnaie nouvelle.

Le Congrès affirme que cette création nouvelle de monnaie ne serait pas inflationnaire puisque l'économie n'opère pas à pleine capacité et qu'elle serait temporaire et limitée à cause des effets conjugués du multiplicateur et de l'accélérateur. Cette dernière remarque suggère qu'il s'agirait de donner à l'économie «la chiquenaude initiale » qui lui permettrait - sans chiquenaude subséquente - de se maintenir à un niveau d'activité qui rétablirait l'équilibre entre l'offre et la demande de situations.

6. Selon le Congrès, il semble qu'il n'existe aucun problème économique à cette suggestion. Non pas qu'il prétende que sa suggestion va sans aucun problème. Mais celui qu'il mentionne en est un d'ordre politique: un bon nombre des projets auxquels le gouvernement affecterait l'argent nouveau relève des juridictions provinciales: il s'agit de dépenses au niveau municipal et de dépenses d'éducation.

7. Les mémoires syndicaux ont souvent l'air d'apporter des solutions évidentes aux problèmes économiques. Et ce n'est pas sans étonnement que l'on se demande comment il se fait qu'on ne les ait pas déjà apportées. Raisons politiques? Inconscience? ou exigences économiques? A la réflexion, on peut trouver des raisons de croire que les deux premiers facteurs jouent. Mais a-t-on des raisons de croire que le troisième ne joue jamais?

Les mécanismes économiques et les données économiques comportent souvent des exigences auxquelles il n'est pas facile d'échapper.

Tout d'abord, les dépenses proposées ne contribueraient pas toutes à augmenter la demande canadienne de produits canadiens. On connaît assez l'importance des importations dans notre économie. Il y aurait donc des fuites.

En second lieu, la partie des investissements qui affecteraient la demande, puis la production canadienne, n’affecterait pas nécessairement l'emploi dans la même mesure.

Le CTC a lui-même présenté à la Commission Gordon un mémoire à cet égard. ${ }^{7}$ Toute augmentation de la production ne se traduit pas nécessairement par une augmentation proportionnelle dans l'emploi. L'augmentation de la production peut porter cette dernière à un niveau

(7) CTC. Effets probables de la mécanisation croissante de l'industrie, Mémoire à la Commission Gordon. 
où l'automation devient possible et frustrer ainsi les espoirs d'une augmentation dans le nombre de situations.

En troisième lieu, il ne suffit pas de déceler, dans l'économie, des facteurs de production non utilisés pour conclure qu'elle n'opère pas à pleine capacité et que toute infusion «limitée et temporaire» de monnaie nouvelle peut ne pas contribuer à l'augmentation des prix. Encore faut-il que tous ces facteurs se présentent dans des proportions telles qu'ils puissent satisfaire aux exigences des coefficients techniques de production. Si tel n'est pas le cas, l'emploi des facteurs de production inutilisés posent des problèmes qui dépassent les cadres de la politique fiscale et monétaire. Pour les employer, il faut trouver les facteurs de production complémentaires - qui eux, peuvent être déjà utilisés à pleine capacité. S'ils sont déjà tous utilisés, on court de grands risques que les dépenses gouvernementales financées à même la création nouvelle de monnaie ne servent qu'à accroître la demande monétaire de ces produits complémentaires qu'on ne peut offrir en plus grande quantité - à moins de s'adresser à l'étranger.

En dernier lieu, il semble difficile de régler un problème structurel, chronique et de longue période par une mesure «limitée et temporaire ». Même s'il était vrai que l'économie n'opère pas à pleine capacité; même s'il était vrai qu’une infusion nouvelle de monnaie réussirait à la porter à un niveau d'opération maximum; même si, à ce niveau, tous les facteurs de production étaient utilisés - il ne s'ensuit pas nécessairement que l'économie se maintiendrait indéfiniment à ce niveau, sans faire appel à d'autres infusions de monnaie «limitées et temporaires». Le CTC laisse cependant entendre qu'après le choc « de la chiquenaude initiale » l'économie atteindrait d'abord son niveau maximum d'opération où tous les individus qui le désirent trouveraient une situation, puis continuerait indéfiniment sur son air d'aller, un peu comme cette boule lancée dans un monde sans friction et qui conserve pour l'éternité sa vitesse initiale...

8. Ces remarques n'impliquent d'aucune façon que la mesure proposée par le CTC n'ait aucun effet sur l'augmentation du nombre de situations. Elles ont pour but, cependant, de signaler qu'il existe des embarras à l'éclosion de ses effets et que ces derniers - comme la mesure qui leur a donné naissance - peuvent être temporaires et limités. Ce n'est d'ailleurs pas la seule mesure proposée par le CTC; mais c'en est une sur laquelle il a beaucoup insisté. Elle offre un moyen d'augmenter l'offre de situations; il en est d'autres, c'est évident. Le CTC en a signalé un certain nombre. Mais il a aussi signalé certaines mesures dont le but était de réduire la demande de situations: augmentation de la période de scolarité obligatoire, réduction des heures de travail.

La première conduit à réduire le nombre de ceux qui se présentent sur le marché du travail; la seconde consiste à « rationner » le travail qu'on offre. Les effets de ces deux mesures ont un caractère de permanence que ceux d'une infusion nouvelle de monnaie peuvent ne pas 
avoir. L'élimination du chômage «structurel, chronique et permanent » ressort beaucoup plus de mesures propres à affecter l'offre de travail (la demande de situations) que de celles propres à affecter la demande de travail (l'offre de situations). ${ }^{8}$

(8) On ne peut passer sous silence l'excellente section du mémoire qui traite du chômage régional. On y fait une revue des mesures prises dans certains pays d'Europe pour y remédier. Nous suggérons nos lecteurs d'y jeter un coup d'oeil.

\section{LA CONTINUITÉ DES OPÉRATIONS DANS L'INDUSTRIE DE LA PULPE ET DU PAPIER}

\section{Jacques St-Laurent}

1. A nouveau, les dirigeants de l'industrie québecoise de la pulpe et du papier posent aux dirigeants des travailleurs la question des opérations continues. "The industry makes no secret that it wants the right to work Sunday ». ${ }^{1}$

2. Tout l'été dernier des dirigeants de l'industrie ont rencontré les dirigeants des unions et des syndicats et les travailleurs eux-mêmes, afin de leur exposer les raisons de cette demande et gagner ces derniers à leur cause. A la suite de ces rencontres et des discussions qu'elles ont soulevées, on a précisé ses positions et pris des décisions. La Fédération nationale des travailleurs de la pulpe et du papier (CSN) a protesté contre tout travail d'opérations le dimanche et a manifesté son intention de s'adresser aux autorités gouvernementales, celles d'Ottawa et de Québec, ainsi qu'aux évêques pour empêcher les compagnies de mettre leur projet à exécution. ${ }^{2}$ D'autre part, Canadian International Paper Co. aurait déjà signé une entente avec l'union locale (CTC) qui représente ses travailleurs à l'effet de travailler le dimanche, si nécessaire, après le ler mai 1961. ${ }^{3}$ On dit aussi que Eastern Canada Newsprint Group, agent négociateur pour plusieurs usines de Québec et de l'Ontario, aurait obtenu, des représentants de leurs travailleurs, un accord de principe. $*$

3. Ce n'est pas la première fois que le problème du travail du dimanche est posé aux travailleurs de l'industrie de la pulpe et du papier. Le 4 mars 1926, le gouvernement Taschereau créait par arrêtéen-conseil une commission d'enquête dont les commissaires « étaient chargés de s'enquérir de la nature et de l'étendue des travaux permis comme urgents et nécessaires dans les usines pour la fabrication de la

(1) Markets Decides New Jobs, The Financial Post, Sept. 17, 1960, p. 11.

(2) Bureau fédéral de la Fédération des Travailleurs de la pulpe et du papier,

Inc., résolution du 23 juillet 1960.

(3) Tough Bargaining Ahead, The Financial Post, id., p. 12.

(4) Idem. 